\title{
A Fast FFT-Based Iterative Algorithm for Image Deblurring With Anti-Reflective Boundary Conditions
}

\author{
Xun Chen ${ }^{\text {a)}}$, Qi Zhu ${ }^{\text {b) }}$ \\ Wuhan Digtal Engineeing Institute, Wuhan 430074 China. \\ a) 7644500@qq.com \\ b) zq xiaop@126.com
}

\begin{abstract}
In this paper we consider the image deploring problem with space invariant PSFs under anti-reflecting boundary conditions. Under the assumption that PSFs are strongly symmetric and boundary values of image are homogeneous, the image deploring problem is transformed to the circular DE convolution problem by odd periodic extension. A fast Landseer iterative algorithm based on the FFT is pro- posed for the 1D and 2D DE convolution problem. In case boundary values of image are nonhomogeneous, due to the anti-reflecting boundary conditions, the bound- ray values of image can be restored by solving a $1 \mathrm{D}$ problem, then the boundary values of image can be homogeneous by subtracting a term related with the re- stored boundary values. If PSFs are not strongly symmetric, the image deploring problem can be transformed to one with strongly symmetric PSFs by re-blurring approach. Numerical experiments show that the efficiency of our algorithm is fast and effective.
\end{abstract}

Key words: PSFs; FFT; Anti-Reflective.

\section{INTRODUCTION}

The digital image deploring is a fundamental issue in digital image process- in. In this paper we consider the classical deploring problem of blurred and noisy images in the case of a space invariant blurring. Image blur is generally modeled as a linear convolution of an image with a Point Spread Function (PSF). The mathematical model of image blurring and noising can be given by the following superposition summation,

$$
\sum_{s=1}^{2 m-1} \sum_{t=1}^{2 n-1} h_{s, t} f_{i+m-s, j+n-t}=g_{i, j}
$$

$(\mathrm{i}, \mathrm{j}) \in\{1, \mathrm{M}\} \times\{1, \mathrm{~N}\}$. Where $\mathrm{f}$ represents an original image, the mask $\mathrm{h}$ with $(2 \mathrm{~m}-1) \times(2 \mathrm{n}-1)$ represents the PSF and $\sum_{s=1}^{2 m-1} \sum_{t=1}^{2 n-1} h_{s, t}=1, g=g^{0}+r$ is the blurred and noisy observed image that is $\mathrm{M} \times \mathrm{N}$ matrix, $g^{0}$ is the blurred image which is acquired by the imaging system and $r$ is the additive noise. Given the PSF h, the image deploring problem is to recover $f$ from the noisy blurred image $g$ in viewable domain $\{1, M\} \times\{1, N\}[1,2]$.

Notice that the blurred image is determined by $f$ in the domain $\{2-\mathrm{m}, \mathrm{M}+\mathrm{m}-1\} \times\{2-\mathrm{n}, \mathrm{N}+\mathrm{n}-1\}$, that is, we have $M N$ equations and $(M+2 m-2)(N+2 n-2)$ unknowns involved. It is clear that the system described by (1) is under-determined since the number of unknowns exceeds the number of equations. In order to cope with this underdetermination, we need some assumptions on $f$ outside the viewable domain, these assumption- $s$ are called boundary conditions. In the image processing literature, some methods have been proposed to assign boundary values, e.g., zero Dirichlet, periodic, reflective, and anti-reflective boundary conditions (see $[3,4,5,6,7]$ and references therein). Zero (Dirichlet) boundary condition assumes that the values of $f$ outside the viewable domain are zero, if the true image is not close to zero at the boundaries, ringing effects will appear in the recon- stricter image due to the introduction of an artificial discontinuity. Periodic boundary condition assumes that values of $f$ outside the viewable domain are exact copies of values inside. In practice, $f$ is commonly not close to periodic, it implies periodic boundary condition 
introduces an artificial discontinuity, ringing effects will also appear. Reflective boundary condition assumes that the scene outside the viewable domain is a reflection of the scene inside the viewable domain, ringing effects are strongly reduced since the artificial boundary discontinuities are eliminated. Anti-reflective boundary condition assumes that the scene outside the viewable domain is the anti-reflection of the scene inside, it has been proposed in [6] and studied in $[8,9$, and 10]. In the noise free case, it has been shown that the anti-reflective boundary condition is the most precise in the sense that no discontinuity in the image or in its normal derivative is artificially added by the imposed boundary condition [6]. Therefore, the ringing effects are negligible with respect to the other boundary conditions [8].

Other boundary conditions have also been proposed to assign boundary values as a local image mean or are obtained by extrapolation methods in or- der to insure continuity. The penalty of their good approximation properties could lie in a linear algebra problem more difficult to cope with.

In this paper, we consider the use of the anti-reflective boundary condition for image deploring. In almost all literature on the image deploring problem under anti-reflecting boundary condition, the large scale ill-conditioned linear system.

$$
\text { Aft }=\mathrm{g}
$$

Arise from (1) is considered. Where $f$ and g are reshaped into vector $f$ and g, coefficient matrix A is defined by the PSF and the anti-reflecting boundary conditions together. Since A is typically severely ill-conditioned, regularizeton is needed in order to compute a stable approximation of the solution of (2). For some classical regularization method (e.g., Tikhonov regularization, CG and Landseer method), it is necessary to compute $A^{T} A$ and $A^{T} \mathrm{~g}$ in the normal equation. In order to overcome the computational difficulties by means of the fast sine transforms (FST), an approach called re-blurring is to replace $A^{T}$ by $A^{\prime}$, where $A^{\prime}$ is the blurring matrix with PSF rotated by 180 degrees, see [8]. The re-blurring approach is relating to the algebra of matrices arising from the imposition of anti-reflecting boundary conditions. While the numerical results appear to support this approach, the theoretical properties remain dubious because the matrix $A^{T} A$ is no normal. As of today, neither a convergence nor a stability analysis appears to be in reach [9]. In [9] a differ- Ent approach is developed to compute regularized solutions of (2), namely by transforming the problem to homogeneous boundary values, and using the fast sine transform to solve the transformed problem. This approach allows to incorporate the known regularization in a very straightforward way but is restricted to strongly symmetric PSFs.

As opposed to the above mentioned (2), we transform the image de- blurring problem to circular DE convolution problem, thus the sophisticated structure of the matrix can be avoided, the fast-trigonometric transforms and the properties of circular convolution can be straightway used, and regular- inaction is easy to incorporate. We learn from the idea in [8], by re-blurring blurred image, our approach is applicable even if PSF is weakly symmetry- rich or nonsymmetrical. It is known that DE convolution problem is ill-posed and regularization theory is often used to solve ill-posed or ill-conditioned.

Problems. For large scale inverse problems, regularized solutions are type- call constructed by iterative regularization method $[11,12,13$, and 14]. Iterative methods generally can be implemented using less storage than direct meth- odds and are often less sensitive to ill-conditioning [15]. Iterative algorithms are very well suited to DE convolution problem. The simplest iterative tech- unique in this class is the Landseer method, but a main disadvantage of the Landseer method is that a large number of iterations have to be performed in order to guarantee the optimal convergence rates. To overcome this probe- elm, a certain acceleration strategy is proposed thanks to the good properties of trigonometric transforms and circular convolution.

In section 2 we transform (1) to circular convolution problem under the assumption that PSFs are strongly symmetric. We show that the bound- ray values of image can be restored by solving four 1D circular convolution problem, and then the boundary values of image can be homogeneous by subtracting a term related with the restored boundary values, the deplored image can be restored by solving a 2D circular convolution problem. In sec- ton 3 a fast Landseer iterative algorithm based on the FFT is proposed for the 1D and 2D convolution problem. Our approach is applicable even if PSF is weakly symmetric or nonsymmetrical by re-blurring blurred image. In section 4 numerical experiments are presented.

\section{RESTORATION OF IMAGE AND CIRCULAR DECONVOLUTION PROBLEM}

Since the PSFs are usually symmetric, see [1], in this paper, first we assume that $\mathrm{h}$ is symmetric, i.e.

$$
h_{s, t}=h_{2 m-s, t}=h_{s, 2 n-t}=h_{2 m-s, 2 n-t}, \quad(s, t) \in\{1, \cdots, m\} \times\{1, \cdots, n\}
$$


In this section, under anti-reflecting boundary conditions and we first extend image $f$ on $Z^{2}$. Let $F$ with size (2M $-2) \times(2 \mathrm{~N}-2)$ is the anti-reflective extension of,

$$
F_{i j}= \begin{cases}f_{i, j}, & (\mathrm{i}, \mathrm{j}) \in\{1, \ldots, \mathrm{M}\} \times\{1, \ldots, \mathrm{N}\}, \\ 2 f_{i, N}-f_{i, 2 N-j} & (\mathrm{i}, \mathrm{j}) \in\{1, \ldots, \mathrm{M}\} \times\{\mathrm{N}+1, \ldots, 2 \mathrm{~N}-2\}, \\ 2 f_{M, j}-f_{2 M-i, j} & (\mathrm{i}, \mathrm{j}) \in\{\mathrm{M}+1, \ldots, 2 \mathrm{M}-2\} \times\{1, \ldots, \mathrm{N}\}, \\ 4 f_{M, N}-2 f_{2 M-i, N}-2 f_{M, 2 N-j}+f_{2 M-i, 2 N-j} & (i, j) \in\{M+1, \ldots, 2 M-2\} \times\{N+1, \ldots, 2 N-2\} .\end{cases}
$$

Further let $\tilde{F}$ denote the anti-reflective extension onZ ${ }^{2}$,

$$
\begin{gathered}
F_{i+k(2 M-2), j+l(2 N-2)}=F_{i, j}+2 k\left(F_{M, j}-F_{1, j}\right)+2 l\left(F_{i, N}-F_{i, 1}\right)+4 k l\left(f_{M, N}-f_{1, N}-f_{M, 1}+f_{1,1}\right), \\
(i, j) \in\{1, \ldots, 2 M-2\} \times\{1, \ldots, 2 N-2\}, k, l \in \text { Z.For all } i, j, k, l \in Z, \\
\left\{\begin{array}{l}
\tilde{F}(1+k(M-1)+i, j)+\widetilde{F}(1+k(M-1)-i, j)=2 \tilde{F}(1+k(M-1), j) \\
\tilde{F}(i, 1+l(N-1)+j)+\widetilde{F}(i, 1+l(N-1)-j)=2 \tilde{F}(i, 1+l(N-1))
\end{array}\right.
\end{gathered}
$$

Clearly equation (1) can be written as:

$$
\sum_{s=1}^{2 m-1} \sum_{t=1}^{2 n-1} h_{s, t} \tilde{F}_{i+m-s, j+n-t}=g_{i, j}
$$

If $(i, j) \in\{M+1, \ldots, 2 M-2\} \times\{1, \ldots, N\}$, From (3) we have,

$$
(i, j) \in\{1, \ldots, M\} \times\{1, \ldots, N\} .
$$

Noticed that $h$ is symmetric,

$$
\widetilde{F}_{i+m-s, j+n-t}=2 \widetilde{F}_{M, j+n-t}-\widetilde{F}_{2 M-i-m+s, j+n-t}
$$

$$
\begin{aligned}
\sum_{s=1}^{2 m-1} \sum_{t=1}^{2 n-1} h_{s, t} \tilde{F}_{i+m-s, j+n-t} & =\sum_{s=1}^{2 m-1} \sum_{t=1}^{2 n-1} h_{s, t}\left(2 \tilde{F}_{i+m-s, j+n-t}-\tilde{F}_{2 M-i-m+s, j+n-t}\right) \\
& =\mathrm{gM}, \mathrm{j}-\sum_{\substack{2 m-1 \\
2 m-1}}^{2 n-1} h_{t=1}^{2 n-1} h_{2 m-s, t} 2 \tilde{F}_{2 M-i-m+s, j+n-t} \\
& =\mathrm{gM}, \mathrm{j}-\sum_{s=1}^{2 n} \sum_{t=1}^{2 n} h_{s^{\prime}, t} \tilde{F}_{2 M-i+m-s^{\prime}, j+n-t} \\
& =\mathrm{gM}, \mathrm{j}-\mathrm{g} 2 \mathrm{M}_{-\mathrm{i}, \mathrm{j}}
\end{aligned}
$$

Similarly, we have:

$$
\sum_{s=1}^{2 m-1} \sum_{t=1}^{2 n-1} h_{s, t} \tilde{F}_{i+m-s, j+n-t}=G_{i, j}, \quad(i, j) \in\{1, \ldots, 2 M-2\} \times\{1, \ldots, 2 N-2\}
$$

Where $\mathrm{G}$ with size $(2 M-2) \times(2 N-2)$ is the anti-reflective extension of $\mathrm{g}$,

$$
G_{i j}= \begin{cases}g_{i, j} & (\mathrm{i}, \mathrm{j}) \in\{1, \ldots, \mathrm{M}\} \times\{1, \ldots, \mathrm{N}\}, \\ 2 g_{i, N}-g_{i, 2 N-j} & (\mathrm{i}, \mathrm{j}) \in\{1, \ldots, \mathrm{M}\} \times\{\mathrm{N}+1, \ldots, 2 \mathrm{~N}-2\}, \\ 2 g_{M, j}-g_{2 M-i, j} & (\mathrm{i}, \mathrm{j}) \in\{\mathrm{M}+1, \ldots, 2 \mathrm{M}-2\} \times\{1, \ldots, \mathrm{N}\} \\ 4 g_{M, N}-2 g_{2 M-i, N}-2 g_{M, 2 N-j}+g_{2 M-i, 2 N-j} & (i, j) \in\{M+1, \ldots, 2 M-2\} \times\{N+1, \ldots, 2 N-2\}\end{cases}
$$

By (5) and definition of $\tilde{F}$,

$$
\sum_{s=1}^{2 m-1} \sum_{t=1}^{2 n-1} h_{s, t} \widetilde{F}_{i+m-s, j+n-t}=\tilde{G}_{i, j}, \quad \mathrm{i}, \mathrm{j} \in \mathrm{Z}
$$

Where $\widetilde{\mathrm{G}}$ is the anti-reflective extension on $Z^{2}$.

\section{Restoration of Boundary Values and 1d Circular DE Convolution Problem}

In this subsection we will show that boundary values off can be restored by solving $1 \mathrm{D}$ circular convolution problems.

In (6), let $i=1$, note that 
And

$$
\widetilde{F}_{1-m-s, j+n-t}+\widetilde{F}_{1+m-s, j+n-t}=2 \widetilde{F}_{1, j+n-t}
$$

We obtain:

$$
\sum_{s=1}^{2 m-1} \sum_{t=1}^{2 n-1} h_{s, t} \tilde{F}_{i+m-s, j+n-t}=\sum_{s=1}^{2 m-1} \sum_{t=1}^{2 n-1} h_{s, t} \tilde{F}_{i-m+s, j+n-t}
$$

$$
\sum_{t=1}^{2 n-1} h_{t}^{a} \tilde{F}_{1, j+n-t}=\tilde{G}_{i, j}, \quad \mathrm{j} \in \mathrm{Z}
$$

Where $h_{t}^{a}=\sum_{s=1}^{2 m-1} h_{s, t}$. By the same reasoning as the derivation of (7) and $\sum_{t=1}^{2 n-1} h_{t}^{a}=1$

Let

$$
\mathrm{f}_{1,1}=\mathrm{g}_{1,1}, \quad \mathrm{f}_{1, \mathrm{~N}}=\mathrm{g}_{1, \mathrm{~N}} \text {. }
$$

And

$$
F_{j}^{u}=F_{1, j}-\frac{f_{1, N-} f_{1,1}}{N-1}(\mathrm{j}-1), \quad G_{j}^{u}=G_{1, j}-\frac{g_{1, N-} g_{1,1}}{N-1}(\mathrm{j}-1), \quad j \in\{1, \ldots, 2 N-2\}
$$

$$
\tilde{F}_{j}^{u}=\tilde{F}_{1, j}-\frac{f_{1, N-} f_{1,1}}{N-1}(\mathrm{j}-1), \quad \tilde{G}_{j}^{\bar{u}}=\tilde{G}_{1, j}-\frac{g_{1, N-} g_{1,1}}{N-1}(\mathrm{j}-1), \quad j \in Z
$$

It is easy to verify that $\widetilde{F}^{u}$ and $\widetilde{G}^{u}$ are odd periodic signals of period $2 N-2$.

Since $h^{a}$ is symmetric,

As a result,

$$
\sum_{t=1}^{2 n-1} h_{t}^{a}(j+n-t)=\sum_{t=1}^{2 n-1} h_{2 n-t}^{a}(j+n-t)=\sum_{t=1}^{2 n-1} h_{t}^{a}(j-n+t)
$$

Accordingly, we have:

$$
\sum_{t=1}^{2 n-1} h_{t}^{a}(j+n-t)=j
$$

$$
\sum_{t=1}^{2 n-1} h_{t}^{a} \tilde{F}_{1, j+n-t}^{u}=\tilde{G}_{i, j}^{u}, \quad \mathrm{j} \in \mathrm{Z},
$$

Neatly, ha is zero padded to Ha have the same size of $F^{u}$,

$$
H_{t}^{a} \begin{cases}h_{n+1-t}^{a}, & t \in\{1, \ldots, n\} \\ h_{2 N+n-1-t,}^{a} & t \in\{2 \mathrm{~N}-\mathrm{n}, \ldots, 2 \mathrm{~N}-2\} \\ 0, & \text { else. }\end{cases}
$$

Let the symbol $\otimes$ denote the circular convolution operator, we consider the circular convolution of $H^{a}$ and $F^{u}$,

Note that

$$
H^{a} \otimes F^{u}=\sum_{t=1}^{2 N-2} H_{t}^{a} \tilde{F}_{j-\mathrm{t}+1}^{u}, \quad j \in\{1, \ldots, 2 N-2\}
$$

And by (8), we have

$$
\begin{aligned}
\sum_{t=1}^{2 N-2} H_{t}^{a} \tilde{F}_{j-\mathrm{t}+1}^{u} & =\sum_{t=1}^{n} h_{n+1-t}^{a} \tilde{F}_{j-\mathrm{t}+1}^{u}+\sum_{t=2 N-n}^{2 N-2} h_{2 N+n-1-t}^{a} \tilde{F}_{j-\mathrm{t}+1}^{u} \\
& =\sum_{t=1}^{n} h_{t}^{a} \tilde{F}_{j-\mathrm{n}+\mathrm{t}}^{u}+\sum_{t=n+1}^{2 n-1} h_{t}^{a} \tilde{F}_{j-2 \mathrm{~N}-\mathrm{n}+2+\mathrm{t}}^{u} \\
& =\sum_{t=1}^{2 n-1} h_{t}^{a} \tilde{F}_{j-\mathrm{n}+\mathrm{t}}^{u}
\end{aligned}
$$

$$
H^{a} \otimes F^{u}=G^{u}
$$

Now we first obtain $F^{u}$ by solving 1D circular convolution problem (9), next obtain the restored boundary values $f_{1, j}$ by the back transformation 


$$
F_{1, j}=F_{j}^{u}+\frac{f_{1, N-} f_{1,1}}{N-1}(\mathrm{j}-1), \quad j \in\{1, \ldots, N\}
$$

Similarly, the other boundary values off can be restored by the same method.

\section{Restoration of Image and 2D Circular DE Convolution Problem}

Once the restored boundary values of $\mathrm{f}$ be obtained, we will show that the image deploring problem can be transformed to 2D circular convolution problem.

Let

And

$$
p_{i, j}^{f}=\frac{\tilde{F}_{M, j}-\tilde{F}_{1, j}}{M-1}(i-1)+\tilde{F}_{1, j}, \quad Q_{i, j}^{f}=\frac{p_{i, j}^{f}-p_{i, 1}^{f}}{N-1}(\mathrm{j}-1)+p_{i, 1}^{f},
$$

It is easy to verify

$$
p_{i, j}^{g}=\frac{\tilde{G}_{M, j}-\tilde{G}_{1, j}}{M-1}(i-1)+\tilde{G}_{1, j}, \quad Q_{i, j}^{g}=\frac{p_{i, j}^{g}-p_{i, 1}^{g}}{N-1}(\mathrm{j}-1)+p_{i, 1}^{g},
$$

And

$$
\tilde{F}^{0}=\tilde{F}-Q^{f}
$$

$$
\tilde{G}^{0}=\tilde{G}-Q^{g}
$$

Becomes odd periodic about $i$ or $j$. Due to symmetry of $h$, we have

Hence, by (6),

$$
\sum_{s=1}^{2 m-1} \sum_{t=1}^{2 n-1} h_{s, t} Q_{i+m-s, j+n-t}^{f}=Q_{i, j}^{g}, \quad i, j \in Z
$$

$$
\sum_{s=1}^{2 m-1} \sum_{t=1}^{2 n-1} h_{s, t} \tilde{F}_{i+m-s, j+n-t}^{f 0}=\widetilde{G}_{i, j}^{0}, \quad i, j \in Z
$$

$F^{0}=\tilde{F}^{0}(1: 2 M-2,1: 2 N-2), G^{0}=\tilde{G}^{0}(1: 2 M-2,1: 2 N-2)$,

And $\mathrm{h}$ is zero padded to $H$ have the same size of $F$,

$$
G_{i j}= \begin{cases}h_{m+1-s, n+1-t}, & (s, t) \in\{1, \ldots, m\} \times\{1, \ldots, n\}, \\ h_{2 M+m-1-s, n+1-t} & (s, t) \in\{2 M-m, \ldots, 2 M-2\} \times\{1, \ldots, n\}, \\ h_{m+1-s, 2 N+n-1-t}, & (s, t) \in\{1, \ldots, m\} \times\{2 N-n, \ldots, 2 N-2\}, \\ h_{2 M+m-1-s, 2 N+n-1-t}, & (s, t) \in\{2 M-m, \ldots, 2 M-2\} \times\{2 N-n, \ldots, 2 N-2\} \\ 0, & \text { else }\end{cases}
$$

For the circular convolution of $H$ and $F^{0}$,

As $\tilde{F}^{0}$ is periodic,

$$
H \otimes F^{0}=\sum_{s=1}^{2 M-2} \sum_{t=1}^{2 N-2} H_{s, t} \tilde{F}_{i-s+1, \mathrm{j}-\mathrm{t}+1}^{u}, \quad i, j \in\{1, \ldots, 2 M-2\} \times\{1, \ldots, 2 N-2\}
$$

Thus, by (10) we obtain:

$$
H \otimes F^{0}=\sum_{s=1}^{2 m-1} \sum_{t=1}^{2 n-1} H_{s, t} \tilde{F}_{i+m-s, j+n-t}^{0}
$$

$$
H \otimes F^{0}=G^{0}
$$

Similar to the restoration of boundary values, we first obtain $F^{0}$ by solving $2 \mathrm{D}$ circular convolution problem (10), next obtain the restored image $\mathrm{f}$ by the back transformation

$$
\begin{gathered}
F_{i, j}=\mid F_{i, j}^{0}+Q_{i, j}^{f} \\
(i, j) \in\{1, \ldots, M\} \times\{1, \ldots, N\} .
\end{gathered}
$$




\section{A FAST LANDWEBER-LIKE ITERATIVE ALGORITHM FOR CIRCULAR DECONVOLUTION}

In the above section, we transform image deploring problems to circular DE convolution problems. As mentioned in Section 1, circular DE convolution problems are typically ill-posed, regularization is needed in order to compute stable approximation of the solution. Landseer iterative method is very well suited to DE convolution problem, but its main disadvantage is that con- mergence may be very slow in practical applications. In this section, in order to overcome this disadvantage of the Landseer method, we apply a fast Landseer-like iterative method proposed in [16] to circular DE convolution problems, and give the corresponding algorithm based on the good properties of trigonometric transforms and circular convolution.

Now we consider general linear ill-posed problems:

$$
T_{x}=y,
$$

Where $T: \mathbb{X} \rightarrow \mathbb{Y}$ is a linear compact operator and $\mathbb{X}, \mathbb{Y}$ are real Hilbert space with corresponding inner product $(\cdot, \cdot)$ and norms $\|\cdot\|$.

In practice, instead of (12) we usually have a perturbed version of the equation

$$
T_{X}=\mathrm{y}_{\mathcal{E}}
$$

Assume $\left(\mu_{\mathrm{j}}, x_{\mathrm{j}}, \mathrm{y}_{\mathrm{j}}\right)$ is singular system of $T$, define $\left(T^{*} T\right)^{\frac{1}{2}}: \mathbb{X} \rightarrow \mathbb{Y}$

Define operator $R_{k}: D\left(T^{\dagger}\right) \rightarrow X$,

$$
\left(T^{*} T\right)^{\frac{1}{2}} z=\sum_{j}\left|\mu_{\mathrm{j}}\right|\left(z, x_{\mathrm{j}}\right) x_{\mathrm{j}}, \quad z \in \mathbb{X}
$$

$$
R_{k}=\varpi\left[\sum_{j=0}^{k-1}\left(I-\left(\varpi T^{*} T\right)^{\frac{1}{2}}\right)^{j}\right]^{2} T^{*}
$$

Where $0<\varpi \leq \frac{1}{\left\|T^{*} T\right\|}$ is relaxation factor, $T^{\dagger}$ denotes Moore-Penrose generalized inverse of $T$, and $k$ rule as regularization parameter. $R_{k y \mathcal{E}}$ Is approximation of $T^{\dagger} y$. The a-posteriori choice of $k$ can be given by Molotov's discrepancy principle, it is that $\mathrm{k}$ is chosen to make $\left\|T R_{k y \delta-y_{\varepsilon}}\right\| \leq \tau \varepsilon$ occur for the first time, $\tau>1$. The regularization solution $R_{k y \varepsilon}$ can be obtained by the following iterative algorithm.

Algorithm 1:

1) Let $_{1}=I, k=1$;

2) $k=k+1$, compute $S_{k}=I+\left(I-\left(\varpi T^{*} T\right)\right)^{\frac{1}{2}} S_{k-1}$;

3) compute $R_{k y \varepsilon}=s_{k}^{2}\left(\varpi T^{*} y_{\varepsilon}\right)$, if $\left\|T R_{k y \delta-y_{\varepsilon}}\right\| \leq \tau \varepsilon(\tau>1)$, then stop, or switch to 2$)$.

It is shown in [16] that the asymptotically optimal convergence order of the regularized solution is obtained but the number of iterations reduce greatly.

Next, we use the above-mentioned method to $1 \mathrm{D}$ and 2D circular DE convolution. For the sake of simplicity, we only consider the $2 \mathrm{D}$ circular $\mathrm{DE}$ convolution. Let $\mathrm{T}_{\mathrm{H}}: \mathrm{R}^{(2 \mathrm{M}-2) \times(2 \mathrm{~N}-2)} \rightarrow \mathrm{R}^{(2 \mathrm{M}-2) \times(2 \mathrm{~N}-2)}$ is discrete circular convolution operator,

Then the equation (11) can be written as:

$$
T_{H} Y=H \otimes X, \quad \forall X \in R^{(2 M-2) \times(2 N-2)} .
$$

$$
T_{H} F^{0}=G^{0},
$$

Notice that $H$ is symmetric, the following result is immediate,

$$
T^{*}{ }_{H}=T_{H}
$$

Let' $\odot$ ' denotes the product of corresponding element, the discrete Fourier transform of $X$ is $\tilde{X}=F(X)$, and the inverse discrete Fourier transform of $X$ is $\tilde{X}=F^{-1}(X)$. By utilizing the discrete Fourier transform, we have a fastiterative algorithm for (15):

Algorithm 2:

1) $\operatorname{Let}_{1}=\hat{\delta}, \varpi=1 / \max (\max (\operatorname{abs}(\widehat{H}))), k=1 ;($ where $\hat{\delta}=\operatorname{ones}(2 M-2,2 N-2))$ 
2) $k=k+1$, computeS $\mathrm{k}=\widehat{\delta}+(\widehat{\delta}-\operatorname{abs}(\varpi \widehat{H})) \odot \mathrm{S}_{\mathrm{k}-1}$;

3) computeW $\mathrm{W}_{\mathrm{k}}=\mathrm{S}_{\mathrm{k}} \odot \mathrm{S}_{\mathrm{k}} \odot\left(\varpi \widehat{H} \odot \hat{G}^{0}\right)$, if $\left\|\widehat{H} \odot \mathrm{W}_{\mathrm{k}}-\hat{G}^{0}\right\| \leq 2 \tau \varepsilon(\tau>1)$, goto4), orgoto2).

4) ComputeF $_{\mathrm{k}}^{0}=\mathrm{F}^{-1}\left(\mathrm{~W}_{\mathrm{k}}\right)$.

In algorithm 2, by discrete Fourier transform, discrete convolution can be turned into product of corresponding element in frequency domain, computing burden have been reduced. Notice symmetry of $\widehat{H}$ and $\widehat{G}^{0}$, the iterative algorithm 2 for $\widehat{H} * \mathrm{~F}=\mathrm{G}$ can be further improved by discrete sine and cosine transforms.

Remark 1: In the case PSF is nonsymmetrical, we will show that the restoration problem can be converted to one with symmetric PSF by re-blurring [8]. If PSF is only weakly symmetric, i.e.

$$
h_{s, t}=h_{2 m-s, 2 n-t}, \quad(s, t) \in\{1, \ldots, m\} \times\{1, \ldots, n\},
$$

Let $h_{1}$ be left-right flipped PSF of $h$, by re-blurring $g$ in (1), we have

$$
\sum_{s=1}^{4 m-3} \sum_{t=1}^{4 n-3}\left(h_{1} * h\right)_{s, t} f_{i+2 m-1-s, j+2 n-1-t}=\tilde{g}_{i, j}
$$

$(i, j) \in\{1, \ldots, M-2 m+2\} \times\{1, \ldots, N-2 n+2\}$ Where $h_{1} * h$ with size $(4 m-3) \times(4 n-3)$ is the linear convolution between $h_{1}$ and $h$, and $\tilde{g}$ is the image re-blurred by PSF $h_{1}$. Clearly, $h_{1} * h$ is symmetric. If PSF is not weakly symmetric, let $h_{1}$ be 180 degrees rotation of $h$, then $h_{1} * h$ is weakly symmetric. In any case, the assumption is appropriate that the PSF is symmetric.

\section{NUMERICAL EXPERIMENTS}

In order to illustrate the efficiency of our algorithm, we consider restoring noisy blurred images $g=g^{0}+r \cdot g^{0}$ represents the blurred image, $r$ is Gaussian white noise.

In our numerical experiments, the original image $f$ with size $256 \times 256$ is the cameraman image from Mat lab (see Figure 1). The restored images are obtained by applying the Algorithm 2 where $\tau=1.1$. the relative error of restored image $f_{k}$ is defined as:

$$
\frac{\|\mathrm{f}-\mathrm{fk}\| \mathrm{F}}{\|\mathrm{f}\| \mathrm{F}} \text {. }
$$

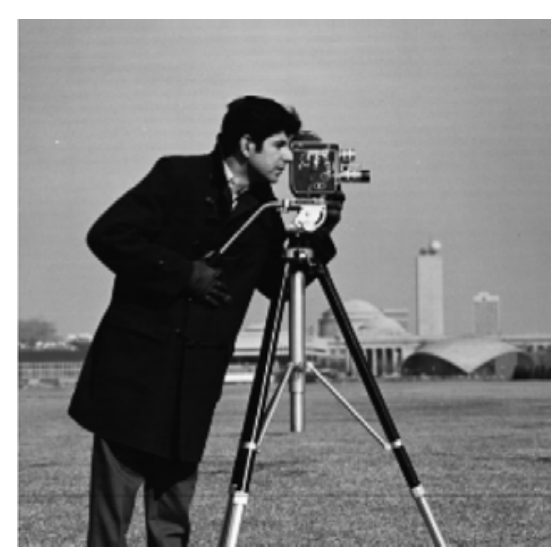

FIGURE 1. Original image.

In the first example, we generated a blurred image $\mathrm{g}$ with size $246 \times 246$ by Gaussian blur with standard deviation 2 and size $11 \times 11$. A Gaussian noise with different levels is added to the blurred image, relative restoration

Errors and the CPU-times are shown in Table 1. The noisy blurred image and restored image are shown in Figure $2-4$.

TABLE 1. Result for Gaussian blur example.

\begin{tabular}{ccc}
\hline noise level & relative restoration error & CPU-time (in seconds) \\
\hline $0.10 \%$ & 0.0935 & 0.828 \\
$1 \%$ & 0.1108 & 0.594 \\
$5 \%$ & 0.1326 & 0.453 \\
\hline
\end{tabular}



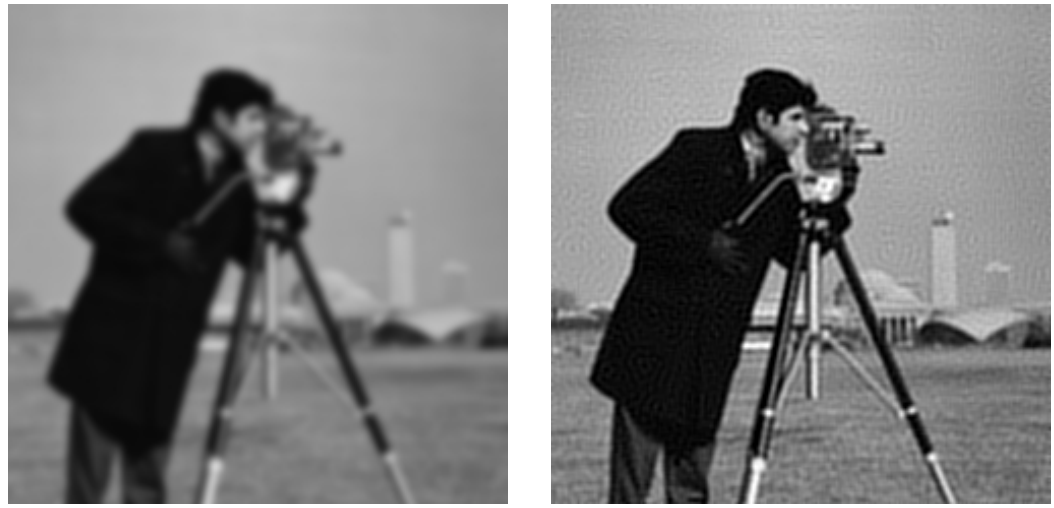

FIGURE 2. Noisy blurred image and restored image for Gaussian blur example with noise level $0.1 \%$.
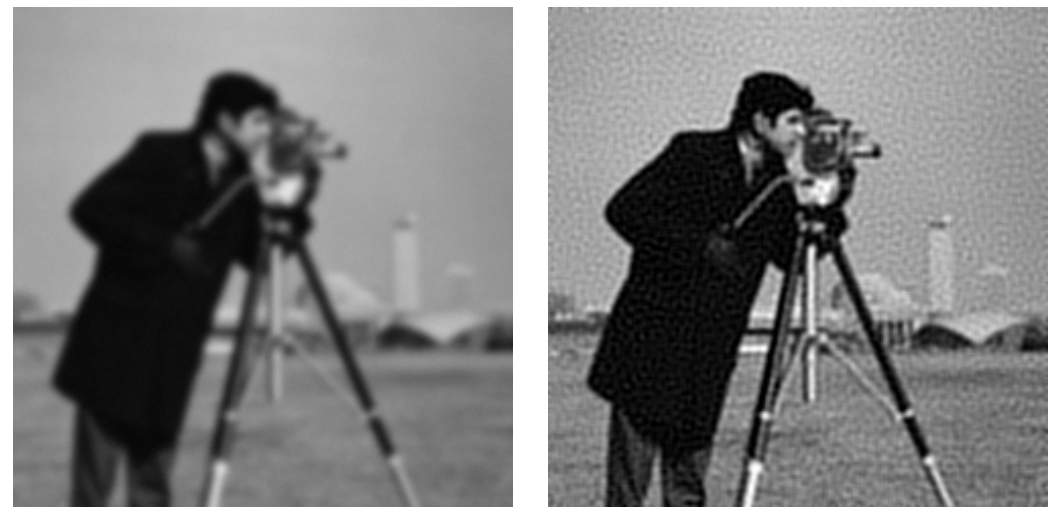

FIGURE 3. Noisy blurred image and restored image for Gaussian blur example with noise level $1 \%$.
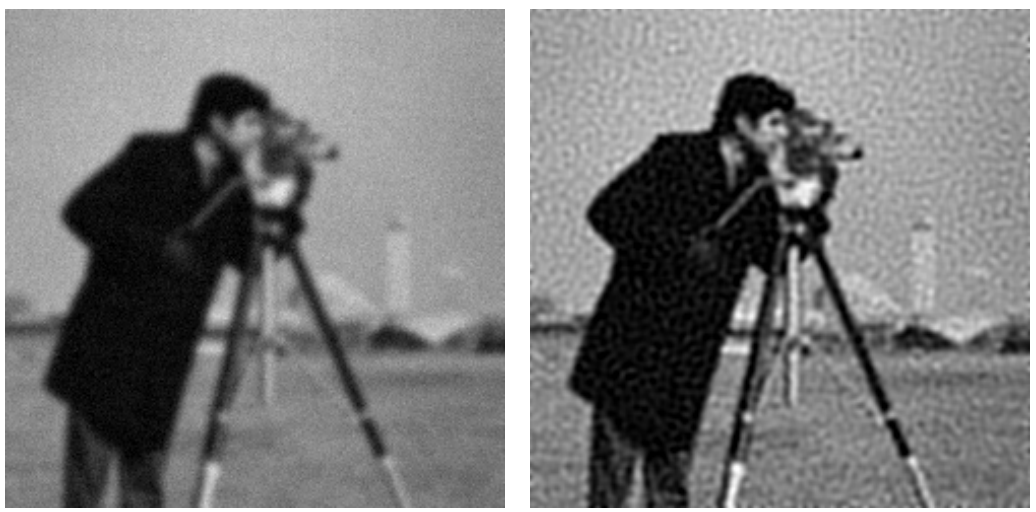

FIGURE 4. Noisy blurred image and restored image for Gaussian blur example with noise level 5\%.

In the second example, we generated a blurred image $g$ with size $246 \times 246$ by out-of-focus blur with radius 5 . A Gaussian noise with different levels is added to the blurred image, relative restoration errors and the CPU-times are shown in Table 2. The noisy blurred image and restored image are shown in Figure 5-7. 
TABLE 2. Result for out-of-focus blur example.

\begin{tabular}{ccc}
\hline noise level & relative restoration error & CPU-time (in seconds) \\
\hline $0.10 \%$ & 0.0847 & 0.781 \\
$1 \%$ & 0.1269 & 0.562 \\
$5 \%$ & 0.1483 & 0.437 \\
\hline
\end{tabular}
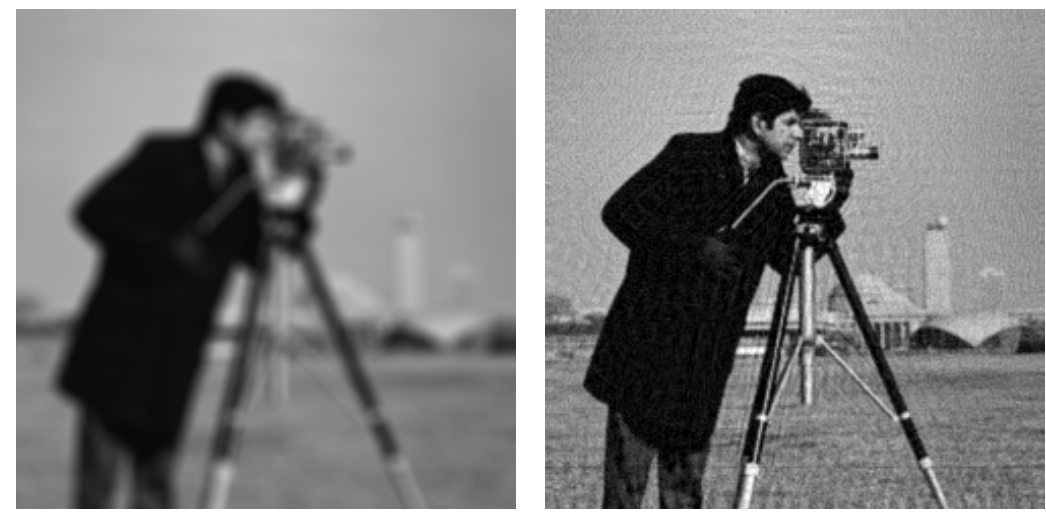

FIGURE 5. Noisy blurred image and restored image for out-of-focus blur example with noise level $0.1 \%$.
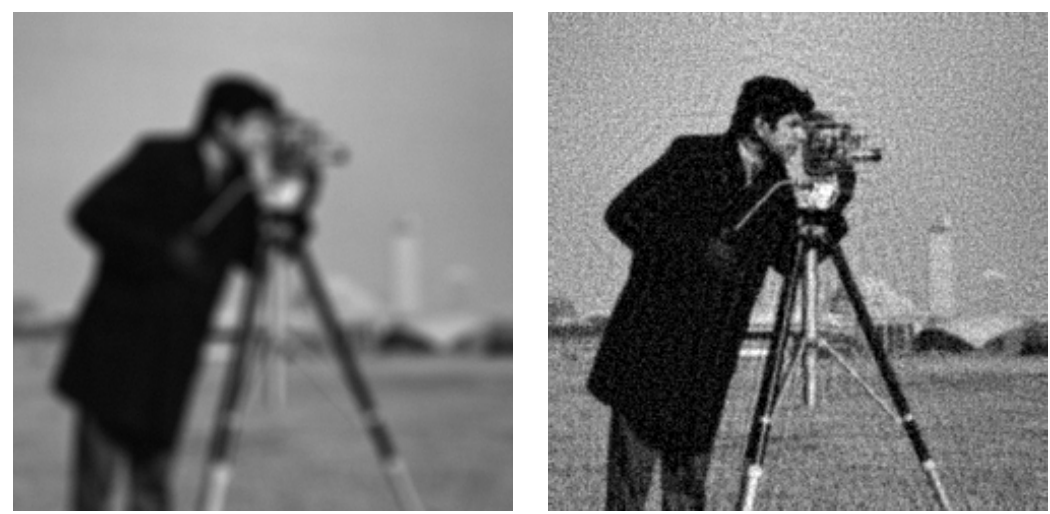

FIGURE 6. Noisy blurred image and restored image for out-of-focus blur example with noise level 1\%.
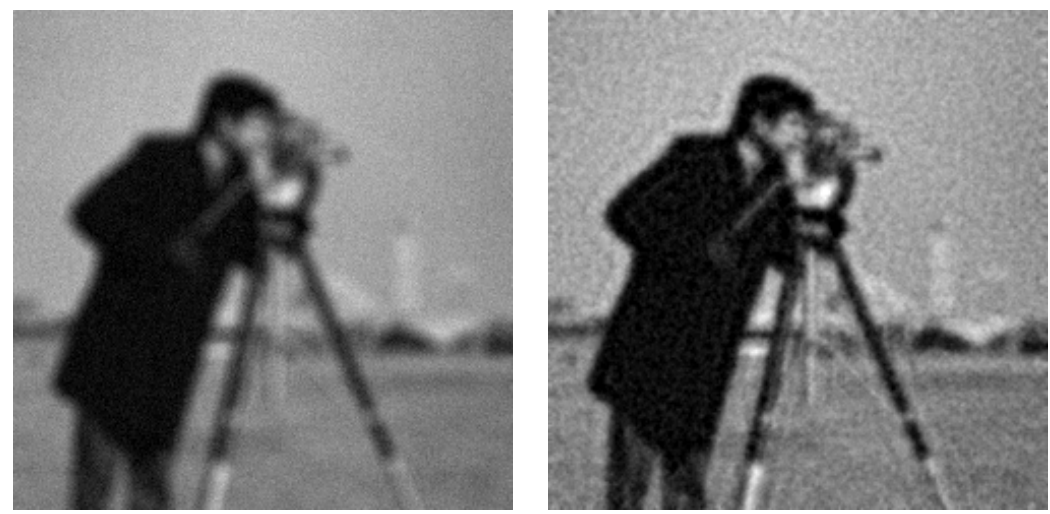

FIGURE 7. Noisy blurred image and restored image for out-of-focus blur example with noise level 5\%. 
In the third example, the PSF is weakly symmetric. We generated a blurred image $\mathrm{g}$ with size $248 \times 248$ by motion blur with 11 pixels and an angle of 45 degrees, $0.1 \%$ of noise is added to the blurred image. Relative restoration error and the CPU-time are shown in Table 3, the blurred image and restored image are shown in Figure 8.

TABLE 3. Result for motion blur example.

\begin{tabular}{ccc}
\hline noise level & relative restoration error & CPU-time (in seconds) \\
\hline $0.10 \%$ & 0.1189 & 1.516 \\
\hline & & \\
\hline
\end{tabular}

FIGURE 8. Blurred image and restored image for motion blur example with noise level $0.1 \%$.

\section{CONCLUSION}

In this paper, under the assumption that the PSF is symmetric, we transform- m image restoration into circular DE convolution problem by using reflecting boundary condition. A fast-convergent method of iterated regularization is applied to DE convolution problem and a fast-iterative algorithm is given by fast discrete Fourier transform. By reblurring blurred image, the algorithm is applicable even if PSF is weakly symmetric or nonsymmetrical. Due to us- in of fast discrete Fourier transform and applying of fast convergent method of iterated regularization, computing burden have been greatly reduced. We remark that the algorithm can be implemented by using discrete cosine trans- form. Numerical results suggest that the algorithm is effective. But we must point out that, if PSF is weakly symmetric or nonsymmetrical, the ill-posedness of DE convolution problem becomes more severe by re-blurring blurred image. It implies that restored image is less effective if PSF is nonsymmetrical.

\section{REFERENCES}

1. A. Jain: Fundamentals of Digital Image Processing, Prentice-Hall, En- Glenwood Cliffs, NJ, 1989.

2. R.L. Lagendijk and J. Bremond: Iterative Identification and Restoration of Images, Kluwer Academic Publishers, Norwell, MA, 1991.

3. H. Andrew and B. Hunt: Digital Image Restoration, Prentice-Hall, En- Glenwood Cliffs, NJ, 1977.

4. R. Gonzalez and R. Woods: Digital Image Processing, Addison-Wesley, Reading, MA, 1992.

5. M.K. Ng, R.H. Chan and W.C. Tang: A fast algorithm for debut- ring models with Neumann boundary conditions, J. Sci. Compute., 21(1999), 851-866.

6. S. Serra-Capizzano: A note on antireflective boundary conditions and fast deploring models, J. Sci. Compute., 25(2003), 1307-1325.

7. Y.Y. Shi and Q.S. Chang: Acceleration methods for image restore- ton problem with different boundary conditions, Appl. Number. Math., 58(2008), 602-614.

8. M. Donatella, C. Static, A. Martin Elli and S. Serra-Capizzano: I'm- proved image deploring with anti-reflective boundary conditions and re-blurring, Inverse Problems, 22(2006), 2035-2053.

9. M. Christiansen, M. Hanker: DE blurring Methods Using Antireflection- tie Boundary Conditions, SIAM Journal on Scientific Computing, 30(2008), 855-872. 
10. M. Donatella, S. Serra-Capizzano: Antireflective Boundary Condition- s for DE blurring Problems, Journal of Electrical and Computer Enki- nearing - Special issue on iterative signal processing in communications, Volume 2010 (2010), 1-18.

11. M. Hanker, A. Neuberger and O. Scherer: A convergence analysis of the Landseer Iteration for nonlinear Illposed problems, J. Number. Math., 72(1995), 21-37.

12. R. Rameau: Modified Landweber Methods for Inverse Problems, Number. Funk. Anal. Optima. 20(1999), 7998.

13. A. Neuberger: On Landseer Iteration for nonlinear Ill-posed problems in Hilbert scales, J. Number. Math., 85(2000), 309-328.

14. P.C. Hansen: Rank-Deficient and Discrete Ill-Posed Problems, SIAM, Philadelphia, 1998.

15. James G. Nagy, Robert J. Clemmons and Todd C. Jorgensen: Tiera- tie image restoration using approximate inverse preconditioning, IEEE Transactions on Image Processing, 5(1996), 1151-1162.

16. X.W. Huang, C.S. Wu and D. Wu: A fast convergent method of iterated regularization, ActiMate. Sci., Ser. B Engl. Ed. 29B (2009), 341-348. 